\title{
METHODS FOR MONITORING THE DETECTION OF MULTI-TEMPORAL LAND USE CHANGE THROUGH THE CLASSIFICATION OF URBAN AREAS
}

\author{
B. I. Alhaddad ${ }^{\text {a, } * \text {, M. C. Burns }}{ }^{\text {a }}$ J. Roca ${ }^{\text {a }}$ \\ ${ }^{\text {a }}$ CPSV, Dept. of Architectonic Construction 1, Polytechnic University of Catalonia (UPC) 08028 Barcelona, Spain - \\ (bahaa.alhaddad, josep.roca, malcolm.burns)@upc.edu
}

Commission IV, WG 8

KEY WORDS: Urban, Classification, Monitoring, Change Detection, Pattern, Segmentation, Contextual, Analysis

\begin{abstract}
:
Urban areas are complicated due to the mix of man-made features and natural features. A higher level of structural information plays an important role in land cover/use classification of urban areas. Additional spatial indicators have to be extracted based on structural analysis in order to understand and identify spatial patterns or the spatial organization of features, especially for man-made feature. It's very difficult to extract such spatial patterns by using only classification approaches. Clusters of urban patterns which are integral parts of other uses may be difficult to identify. A lot of public resources have been directed towards seeking to develop a standardized classification system and to provide as much compatibility as possible to ensure the widespread use of such categorized data obtained from remote sensor sources.

In this paper different methods applied to understand the change in the land use areas by understanding and monitoring the change in urban areas and as its hard to apply those methods to classification results for high elements quantities, dusts and scratches (Roca and Alhaddad, 2005). This paper focuses on a methodology developed based relation between urban elements and how to join this elements in zones or clusters have commune behaviours such as form, pattern, size. The main objective is to convert urban class category in to various structure densities depend on conjunction of pixel and shortest distance between them, Delaunay triangulation has been widely used in spatial analysis and spatial modelling. To identify these different zones, a spatial density-based clustering technique was adopted. In highly urban zones, the spatial density of the pixels is high, while in sparsely areas the density of points is much lower. Once the groups of pixels are identified, the calculation of the boundaries of the areas containing each group of pixels defines the new regions indicate the different contains inside such as high or low urban areas. Multi-temporal datasets from 1986, 1995 and 2004 used to urban region centroid to be our reference in this study which allow us to follow the urban movement, increase and decrease by the time. Kernel Density function used to Calculates urban magnitude, Voronoi algorithm is proposed for deriving explicit boundaries between objects units. To test the approach, we selected a site in a suburban area in Barcelona Municipality, the Spain.
\end{abstract}

\section{INTRODUCTION}

Remote sensing in urban areas has been a challenger for quite some time due to their complexity and fragment with combination of man-made features and natural features. High-resolution satellite images offered potential possibilities for change detection and spatial modelling in urban areas. To understand the dynamics and patterns of urban change related with their interactions in heterogeneous landscapes, the spatial complexity needs to be quantified accurately, depending upon the morphological analysis and their relation with territory. Morphological analysis, which refers to the geometric characteristics of urban sites, illustrates its usefulness in determining the analogies between patterns of cities and their "physical" characters providing indicators of the aspect of settlement form and structure. Remote sensing might be helpful on the regional scale in evaluating the role that landscape play in connecting different settlements within urban regions and in separating the core city from the surrounding countryside. It used to map urban morphology of human settlements and monitoring urban growth (Batty and Longley, 1987). The information produced by remote sensing is spatially referenced through an implicit geometric location of the pixels. Various urban forms are potentially discernible using such devices, including linear objects (Sohn and Bowman, 2001). Monitoring the urban change inside Barcelona municipality in base of pixel-based classification results reflect the combination of complex special artificial areas. To simplify the monitoring of urban change the work consisted basically in the definition of technical criteria to delineate administrative boundaries from Adjacent Urban Objects related to their Shortest Links and Nearest Distance which making easy to cluster, classify, evaluate and compare different between urban zones related to the urban compactness and densities.

The developed work tackles, in the first place, urban objects divided to various regions of structure densities depend on the land occupation area and pixel neighbours distance, each certain region present administrative boundary already converted (each pixel) to Points Of Interest (POIs) to be available for our study in the income sections. In the second place, based on the identification of groups of points, exploits the fact that POIs are geographically distributed in clusters. In highly urban regions, the spatial density of the POIs is high, while in sparsely areas the density of points is much lower. To identify these different regions, a spatial density-based clustering and delineating techniques were adopted. In the third place, it concentrates on the analytical process in relation to centroid point data distributions. Kernel Density function used to calculates a magnitude per cluster area from centroid point features to fit a smoothly tapered surface to each point to monitor Spars Urban Sprawl. In the fourth place,

* Corresponding author. 
it proposes for deriving explicit boundaries between cluster areas from centroid point features to illustrate urban dynamic change presented by the moving centroid cluster points through Dynamic Voronoi method.

Finally the experimental results show that the proposed pixelbased classification approach is powerful for spatial clustering. This research carried out focusing upon the development of a methodology to classify the geometric properties and extract proximity relations among disjoint objects. We expect that the used methods are quite suitable in monitoring the change detection in urban areas.

\section{DATA AND STUDY AREA}

The chosen area is Barcelona, which is the regional capital of Catalonia, lying in the north-east of Spain (see Figure 1). The physical limits of Barcelona extend to almost $100 \mathrm{Km} 2$. and the city had a population of some $1,595,110$ inhabitants in January 2007 leading to a population density of almost 16,000 inhabitants / Km2.

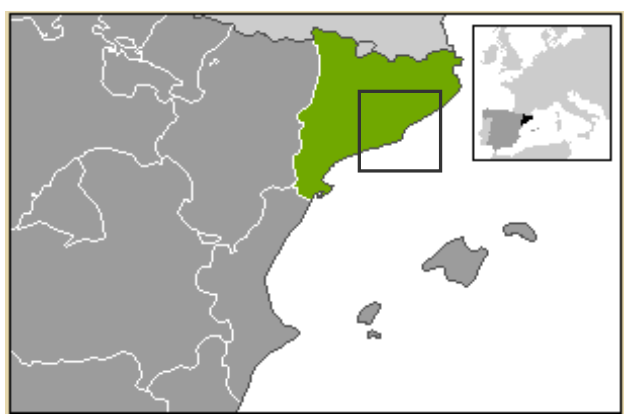

Figure 1: The Autonomous Community of Catalonia.

Three Remote sensing imageries were used for the study. SPOT satellite imagery dating from 1986, 1995 and 2004; in order to quantify and analyze the process of 'peri-urbanization' which has been experienced in the Barcelona city over this period. At the same time examine the increased accuracy for urban planning activities from an operational perspective, afforded by the new generation of satellite images from SPOT5, through the lower and higher resolution of the images, for the monitoring of key urban development issues, both within the confines and beyond the edges of Barcelona's municipality.

\section{METHODOLOGY}

\subsection{Spatial Clustering of Pixels Based on Shortest Links between Adjacent Urban Objects}

Typically the term segmentation describes the process, both human and automatic, that separates zones or regions in an image showing some characteristic with respect to a certain evaluation function (Gelsema and Kanal, 1994). Theses characteristics could be, for example the percent of land occupation in one area and pixel neighbours distance and other characteristics such brightness or colour, roughness, texture. Special homogeneity of pixels plays the most important role in special segmentation, and this is used in our study case. Delaunay triangulation applied to the previous segmented urban areas for finding adjacent areas and the shortest distance between them.
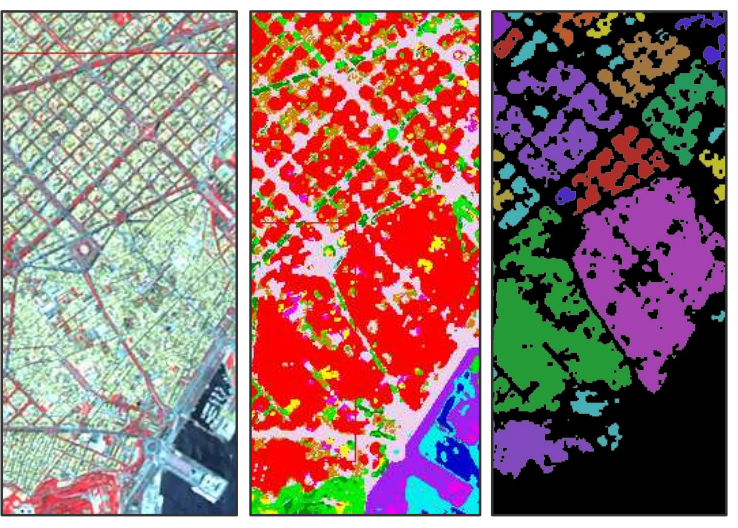

Figure 2: The segmentation result of urban area to following regions size and contents.

To do so, we must eliminate triangle edges that link two pixels of one and the same object. The remaining edges indicate adjacent objects. Thus the shortest edge between two adjacent objects can be extracted for representing the proximity relationship and how close these objects are situated (i.e. proximity). We describe how a set of geographic data can be used to create a space model based on location contexts. The proposed process includes three major stages (see Figure 3).

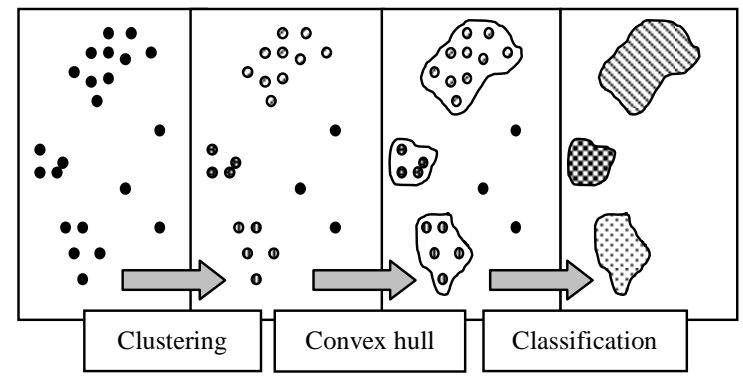

Figure 3: Generating location contexts from POIs

\subsection{Spatial Analysis Using Delaunay Triangulation}

The Delaunay triangulation links up the natural neighbours in a point set by triangle edges; the edges of such triangles indicate the proximity relationship between linked points. If applied to the centres of pixels that represent image objects, we get triangle edges between adjacent pixels of one and the same image object and triangle edges that link up two pixels of adjacent image objects, the two pixels satisfying the natural neighbour criterion. Thus the shortest links between two adjacent image objects can be derived based on the length of the edges that link two adjacent image objects. In raster to vector conversion of the labelled image, the centre of building pixel becomes a point; we use the coordinate number (x,y) of a pixel to represent a point vector, (see Figure 4), and use invent the ID of the point. To extract adjacent image objects, we deploy Delaunay triangulation for all pixels that constitute image objects such as buildings; each triangle edge indicates proximal points (pixels), (see Figure 5). 


\subsection{POIs and morphological image processing}

Classification land use datasets normally carried a lot of noise (seed pixels) which makes difficult to analyze and understand the conjunction and the similarity of zones for urban growth study, morphological images processing will simplified and identify the urban form. Since the spatial presentation of an image object in image space is a binary segment, the region adjacency graph (RAG) can be created based on morphological image analysis.

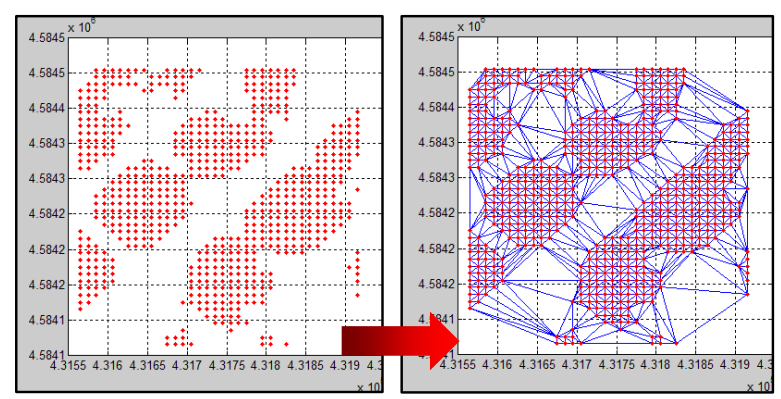

Figure 4: (Up) Pixels (points) embedded by image objects such as buildings, Barcelona city, 2004.

Figure 5: (Down) Delaunay triangulation deployed in all building pixels.

The morphological approach is based on operators such as dilation, erosion, opening, closing etc. Dilation can be used to determine how close adjacent objects are by controlling the repeated application of the dilation operator till various segments merges to one. Clustering is one of the most important morphological analysis tasks. The goal is to divide a collection of objects into groups, such that the similarity between objects in the same group is high and objects from different groups are dissimilar. The natural clusters extracted by this approach are presented in Figure 7. In this experiment, image cluster of urban areas were combined in a single image (see Figure 6) from cluster areas Convex hull already applied to reform urban areas to homogeneous zones (see Figure 8). Centroid point patterns of clustered satellite images from 1986 to 2004 (see Figure 9) examined to understand urban pattern behaviour which is usually broadly classified as random, uniform or clustered, both within the confines and beyond the edges of Barcelona's municipal limits.

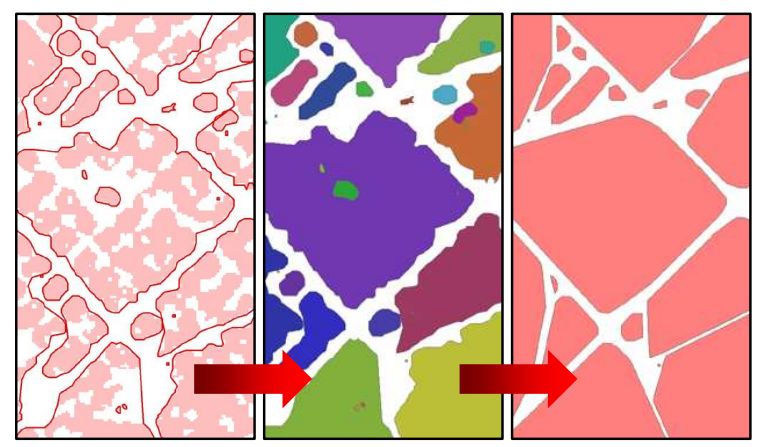

Figure 6: (Left) combined image using image segments.

Figure 7: (Middle) extracted spatial units based on morphological analysis, a raster presentation of clustered blocks.
Figure 8: (Right) reform urban clusters areas using convex hull algorithm.

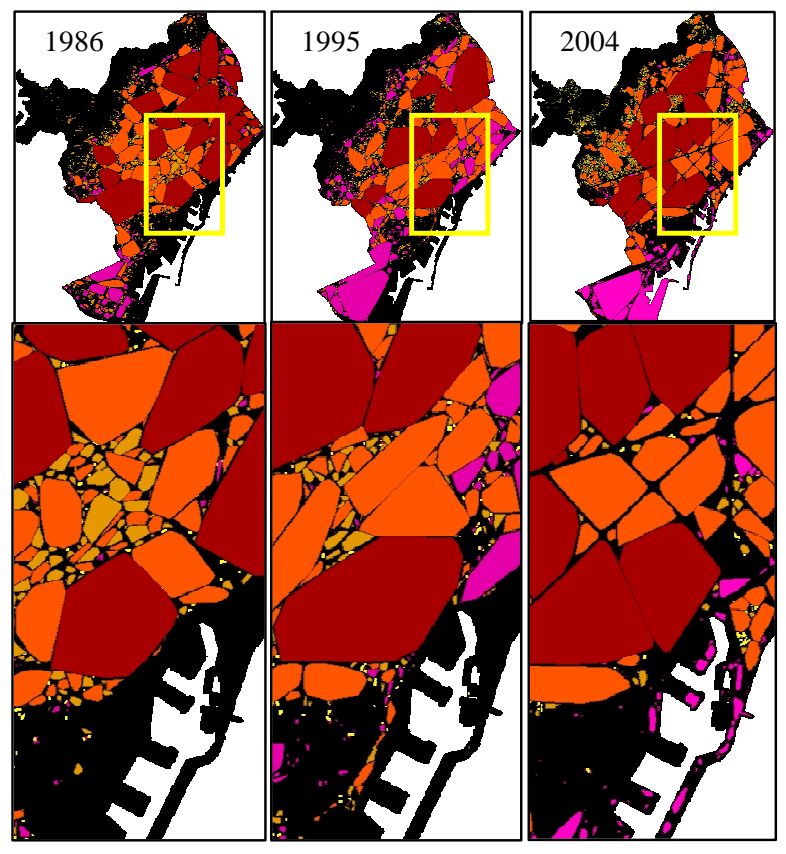

Figure 9: Multi-temporal clustered classification results.

\subsection{Monitoring Sprawl using Smoothing Parameter and Kernel Density}

This section will concentrate on the analytical process in relation to centroid point data distributions. Point pattern events on maps change through time, so an important aspect stressed by many authors (Zeng and Chen, 2006) (Diggle and Chetwynd, 1991) is to try to make the linkage between the spatial map and temporal changes. Figure 10 shows the centroid of joined clustered areas in Barcelona municipality over the period 19862004. Visual inspection suggests that several clusters of different types and sizes exist, but the initial inspection of the mapped data is somewhat misleading. Examining the source dataset shows that roughly the most of the point dataset consists of closest locations, i.e. points are presented closest isolated urban fabric.

Assuming the point set is un-weighted, and exhibits marked clustering, it is then useful to identify factors such as: (1) where are the main (most intensive) clusters located? (2) Are clusters distinct or do they merge into one another? (3) Are clusters associated with some known background variable, such as reflecting variations in land-use (farmland, forest, water etc.)? (4) Is there a common size to clusters or are they variable in size? (5) Do clusters themselves cluster into higher order groupings? (6) If comparable data are mapped over time, do the clusters remain stable or do they move and/or disappear? Again, there are many questions and many more approaches to addressing such questions. An alternative approach to density computation for two-dimensional point-sets is based on techniques developed in one-dimensional (univariate) statistical analysis. The usual way in which we examine patterns of centroide dataset is by smoothing the data to iron out the connectivity that take place from data that is originally represented by urban clusters. The simplest way is to take a moving average of the data which consists of averaging the data 
in a window or neighbourhood defined around each basic location. A more controlled method for achieving such smoothing is by using a kernel density estimator (KDE) such as the one in the proprietary GIS software ArcView (Mitchell, 1999).

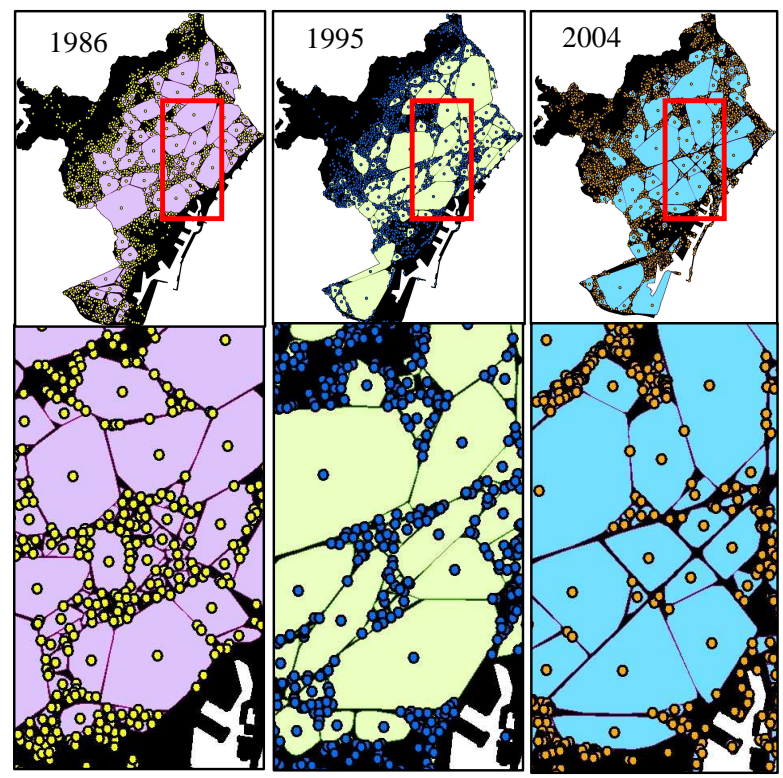

Figure 10: Centroid point dataset corresponding to multitemporal joined cluster areas.

This is based on Silverman's (Silverman, 1986) quadratic and it is used to generate different levels of surface smoothing choosing different sizes of bandwidth - akin to different window sizes. With a specific kernel function, it is the value of the bandwidth, which determines the degree of averaging in the estimate of the density function. The possibility of "manipulating" the results of the KDE through the application of different bandwidths and functions can be empirically translated into testing different assumptions about the spatial behaviour of a particular variable such as its distance decay effects. In Figure 11 the kernel density procedure has been applied to a dataset of above cluster centroide of Barcelona Municipality. Cases are shown as points in this map, with areas of higher-kernel density being shown in darker tones. The highlighted areas in the upper left of the map present lowerkernel density.

\section{URBAN CHANGE DETECTION USING DYNAMIC VORONOI DATA STRUCTURE}

Static point Voronoi tessellations are well known in the literature, and algorithms have been used for many years. Less well known are dynamic algorithms that allow point creation, deletion and movement, and also Voronoi tessellations of more complex objects - typically line segments as well as points (Aurenhammer, 1991). Algorithms for generating the simple point Voronoi tessellation have improved significantly in theoretical efficiency in recent years. However, as a major motivation for this work concerned the maintaining of a map when urban cluster centroides (see Figure 10) are moving by time, an alternative technique was developed that maintained the Voronoi spatial relationships while map objects were being inserted, deleted, or displaced. This is achieved by determining when the Voronoi cell of a moving point gains or loses a neighbouring cell, moving the point to that location, and locally updating the topological structure accordingly.

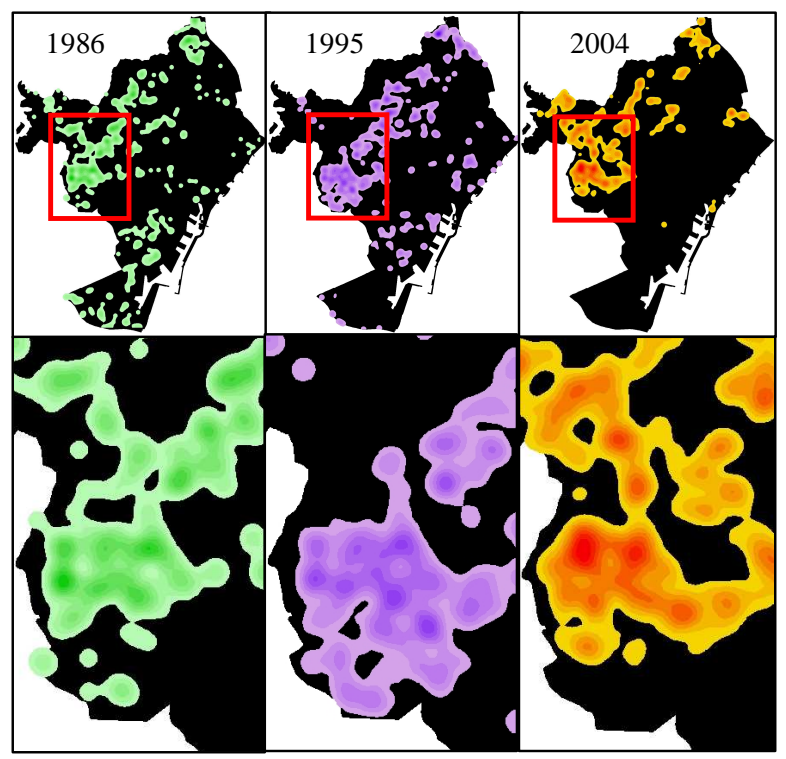

Figure 11: Change of the smoothing parameters of Urban Density. Kernel density with window 10 by 10 meters and $300 \mathrm{~m}$ search radius already applied for above results.

\subsection{Dynamic Voronoi and Interpolation Cluster Centroid Data}

The natural neighbour (Sibson, 1981) (Watson, 1987) method of interpolation is based on one idea: that if the "query point" (at the location where it is desired to make an interpolation estimate- i.e. new centroid of a new urban cluster area) is inserted into the Voronoi tessellation formed by the real data points, then it will reduce ("steal") some area from the adjacent Voronoi cells. These areas are ideally suited as weights to be used for calculating the weighted average of the heights of the neighbouring data points. When the query point coincides with a data point all of the stolen area will be taken from that particular data point's Voronoi cell.

This produces one of the few spatial models that can guarantee that an interpolated surface both passes through all data points and has no discontinuities or other artifacts. Figure 12 shows that the interpolated surface resulting from this is continuous within the convex hull of the data, red colour present the smallest Voronoi cell (spars built-up areas) and the green colour present the biggest Voronoi cell (high or no built-up area), time and urban growth will help each cell to be divide (new built-up areas) for two or more cells with less size, when the cells areas come to be zero the conjunction neighbours will explode to be again one big Voronoi cell illustrating high built-up area.

\section{CALCULATING THE EAREST EIGHBOUR DISTANCE STATISTICS}

The Average Nearest Neighbour Distance tool measures the distance between each feature centroid and its nearest neighbour's centroid location. It then averages all these nearest neighbour distances. If the average distance is less than the average for a hypothetical random distribution, the distribution of the features being analyzed are considered clustered. If the average distance is greater than a hypothetical random distribution, the features are 
considered dispersed. The index is expressed as the ratio of the observed distance divided by the expected distance (expected distance is based on a hypothetical random distribution with the same number of features covering the same total area).

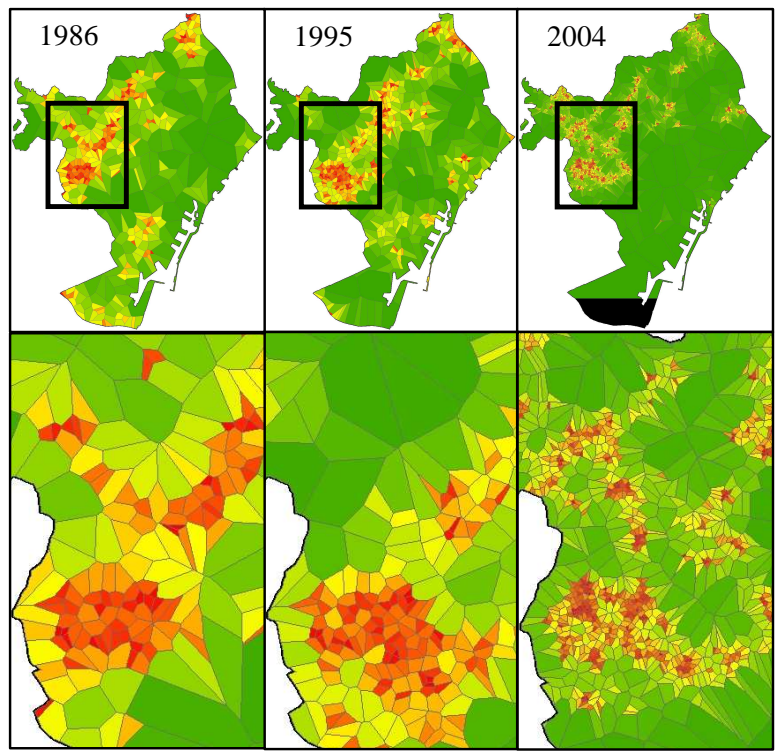

Figure 12: Dynamic Voronoi for multi-temporal map illustrates change detection presented by moving cluster centroid from Voronoi cells.

\section{CALCULATING THE EAREST EIGHBOUR DISTANCE STATISTICS}

The Average Nearest Neighbour Distance tool measures the distance between each feature centroid and its nearest neighbour's centroid location. It then averages all these nearest neighbour distances. If the average distance is less than the average for a hypothetical random distribution, the distribution of the features being analyzed are considered clustered. If the average distance is greater than a hypothetical random distribution, the features are considered dispersed. The index is expressed as the ratio of the observed distance divided by the expected distance (expected distance is based on a hypothetical random distribution with the same number of features covering the same total area). The Nearest Neighbour Distance index can give us an idea whether or not we're dealing with clustered or dispersed data, as it measures the average distance between points in our data and compares the measurement to the expected measurement (a hypothetical random distribution). The Nearest Neighbour Distance index can essentially be boiled down to:

--- (<1-clustered) --- (1-random) --- (>1-dispersed) ---

Scores less than one indicate clustering, and scores higher than one indicate dispersion. Running the tool on our Voronoi datasets, we get the following, Figure 13.

\section{CONCLUSION}

Remotely sensed data products have got unique advantage over conventional data gathering techniques in the study of urban morphology and identify cities structures. The physical parameters like urban form, objects relation and urban structure as well as functional characteristic which can be derived from land use classification, area clearly visible on satellite data products. Remote sensing data is capable of detecting and measuring a variety of elements relating to the morphology of cities, such as the amount, shape, density, distance and spread of urban areas.

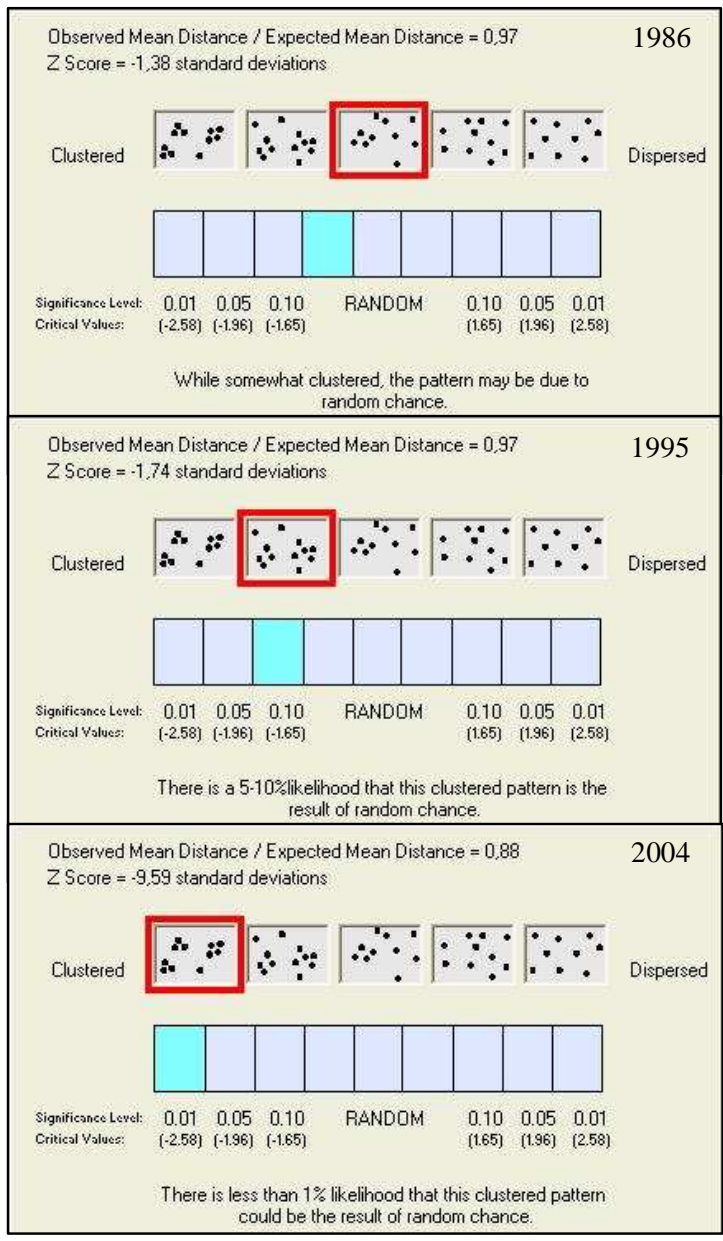

Figure 13: Voronoi nearest neighbour index expressed the ratio of the observed distance divided by the expected distance.

The present study has demonstrated methods for urban areas to identify measure and monitor various patterns of urban behaviour and change in the Barcelona city, by integrating with remote sensing and GIS techniques. The current methods can be easily implemented within GIS to facilitate the measurement of urban growth. Delaunay triangulation has demonstrated a good tool for extracting proximity relations among disjoint objects to natural clusters consist of urban areas that are identified as the nearest neighbours also to classify urban areas in zones easier to follow them impact change by the time. The experimental results and acquired relationships show that the shortest distance between clusters and similarity measures in terms of urban classification segments are good measures. Kernel density estimation (KDE) examined the highest densities of clustered areas to indicate the change. Voronoi diagram examined urban pattern behaviour which is usually broadly classified as random, uniform or clustered, both within the confines and beyond the edges of Barcelona's municipal limits.

\section{REFERENCES}

Batty M., Longley P.A., 1987. Urban Shape as Fractal. Area 19.3, 215-221. 
Bundy G. L., Jones C. B. \& Furse, E. 1995. Holistic generalizetion of large-scale cartographic data. In: J. C. Muller, J. P. Lagrange and R. Weibel (eds.), GIS and Generalization. London etc., Taylor \& Francis: pp.106-119, 1995.

D.F. Watson \& G.M. Philip. 1987. Neighbourhood-based interpolation. Geobyte: vol. 2, pp. 12-16.

Diggle PJ \& Chetwynd AG. 1991. Second-Order Analysis of Spatial Clustering for Inhomogeneous Populations. Biometrics: Vol. 47, No. 3, 1155-1163.

F. Aurenhammer. 1991. Voronoi diagrams - a survey of a fundamental geometric data structure. ACM Computing Surveys 23: $345-405$.

Gelsema E.S., Kanal L.N., 1994. Multiple Paradigms, Comparative Studies and Hybrid Systems. In: Pattern Recognition in Practice IV, Amsterdam, the Netherlands.

Gong, P., Marceau, D. J. \& Howarth, P. J. 1992. A comparison of spatial feature extraction algorithms for land-use classification with SPOT HRV data. Remote Sensing of Environment 40: 137-151.

Haralick, R. M., Shanmugam, K. \& Distein, I. 1973. Textural features for image classification. IEEE Transactions on System, Man, and Cybernetics, SMC-3: 610-621.

J. Roca, M. C. Burns, P. Garcia, Bahaaeddin IZ Alhaddad, R. Colombo and J.M. Carreras, 2005. Reconciling Precision and Scale in Monitoring Urban Land Cover Changes with Satellite Imagery. 3rd International Symposium Remote Sensing and Data Fusion Over Urban Areas, 5th International Symposium Remote Sensing of Urban Areas, Arizona, USA, Vol. XXXVI, Part 8/W27, ISSN 1682-1777.

Marceau, D. J., Howarth, P. J., Dubois, J. M. \& Gratton, D. J. 1990. Evaluation of the grey-level cooccurrence matrix method for land-cover classification using SPOT imagery. IEEE Transactions on Geoscience and Remote Sensing 28: 513-519.

Ma J, Zeng D. \& Chen H. 2006. Spatial-Temporal CrossCorrelation Analysis: A New Measure and a Case Study in Infectious Disease Informatics. In Proceedings of the Intelligence and Security Informatics: IEEE International Conference on Intelligence and Security Informatics (ISI 2006): 23-24. San Diego, CA, USA.

Mitchell, A. 1999. Geographic Patterns \& Relationships. The ESRI Guide to GIS Analysis: Volume 1, Redlands, CA: ESRI Press.

R. Sibson. 1981. A brief description of natural neighbour interpolation. In V. Bamett (ed.), Interpreting Multivariate Data, New York: John Wiley: 2 1- 36.

Silverman, B. W. 1986. Density Estimation for Statistics and Data Analysis. New York: Chapman \& Hall.

Sohn, G., Dowman, I., 2001. Extraction of buildings from high resolution satellite data. In: Baltsavias, E., Gruen, A., Van Gool, L. (Eds.) Automated Extraction of Man-Made Object from Aerial and Space Images (III). Balkema Publishers, Lisse, pp.345-355.

\section{ACKNOWLEDGEMENTS}

The authors of this paper gratefully acknowledge the research funding provided by both the European Commission through the ERDF, by way of the INTERREG IIIB Programme, the Cartographic Institute of Catalonia and the Spanish Ministry of Science and Technology (ref. BIA2003-07176). Thanks to the referees for their help. 